
A.B. Kanwe ${ }^{1}$
Z. Bengaly
D. Saulnier ${ }^{1}$
G. Duvallet ${ }^{1}$

\section{Évaluation du test de détection des antigènes circulants de trypanosomes à l'aide d'anticorps monoclonaux. Infections expérimentales et naturelles}

KANWE (A.B.), BENGALY (Z.), SAULNIER (D.), DUVALLET (G.). Évaluation du test de détection des antigènes circulants des trypanosomes à l'aide d'anticorps monoclonaux. Infections expérimentales et naturelles. Revue Élev. Méd. vét. Pays trop., 1992, 45 (3-4) : 265-271

Un test ELISA de détection des antigènes circulants pour le diagnostic des trypanosomoses animales a été proposé récemment par NANTULYA et LINDQVIST (1989). Basé sur l'utilisation d'anticorps monoclonaux spécifiques, ce test est réputé permettre le diagnostic d'une infection active et l'identification du ou des trypanosomes responsables. Avec les réactifs fournis gracieusement par l'International Laboratory for Research on Animal Diseases (ILRAD), on a pu l'évaluer au CRTA, lors d'infections expérimentales de petits ruminants et sur des sérums de bovins prélevés sur le terrain. De plus, sur 40 sérums de bovins prélevés en France et testés, les densités optiques (DO) de 0,007 à 0,009 ont été obtenues en utilisant les 3 anticorps monoclonaux anti-T. congolense, anti-T. vivax et anti-T. brucei. Ces DO sont bien inférieures à la valeur 0,050 retenue comme seuil de positivité par l'ILRAD. Lors des infections expérimentales de petits ruminants, la sensibilité du test a été de 63,2 p. 100 pour les animaux infectés à $T$. congolense et de $9,9 \mathrm{p} .100$ seulement pour les animaux infectés par T. vivax La sensibilité des tests parasitologiques était respectivement de 55,1 et 48,6 p. 100. La combinaison des deux tests a permis de porter la sensibilité à respectivement 82,4 et 52,8 p. 100 . Concernant les sérums de bovins découverts infectés sur le terrain, les moyennes des DO observées étaient, en réaction homologue, de $0,116 \pm 0,030$ pour $T$. congolense et $0,011 \pm 0,028$ pour $T$. vivax. Parmi les 20 sérums d'animaux infectés à $T$. congolense, 16 ont donné une DO supérieure à 0,050 (sensibilité $80 \mathrm{p} .100$ ) et parmi les 20 sérums d'animaux infectés à $T$, vivax, un seul a donné une DO supérieure à 0,050 (sensibilité 5 p. 100). La recherche d'une valeur seuil de DO inférieure à 0,050 fondée sur l'analyse de sérums pré-infection, ou sur l'étude d'animaux provenant de zones non infectées, permettrait d'augmenter la sensibilité du test. Il faut noter que ces résultats ont été obtenus pendant la phase d'infection aiguë des animaux, notamment pour les infections expérimentales. Or, les résultats obtenus à l'ILRAD montrent que ce test est beaucoup plus efficace pendant les phases chroniques aparasitémiques. D'autres essais sont en cours au CRTA, notamment sur le terrain lors d'enquêtes épidémiologiques. Mots clés : Trypanosoma congolense. Trypanosoma vivax Trypanosoma brucei - Trypanosomose - Diagnostic - Détection d'antigène circulant - Test ELISA - Anticorps monoclonal - Burkina Faso.

\section{INTRODUCTION}

Les trypanosomoses sont toujours une contrainte majeure pour l'élevage dans les zones humides et subhumides d'Afrique au sud du Sahara. Des recherches sont menées dans l'ensemble du continent pour améliorer le diagnostic et la lutte contre ces maladies. Les techniques

1. Centre de recherches sur les trypanosomoses animales (CRTA), 01 BP 454, Bobo-Dioulasso 01, Burkina Faso.

Reçu le 11.8.1992, accepté le 5.2.1993. classiques de diagnostic parasitologique des trypanosomoses sont assez peu sensibles (7). Récemment, il a été montré que la technique de double micro-centrifugation (1) permettait de détecter des parasitémies inférieures à $10^{2}$ parasites $/ \mathrm{ml}$ pour Trypanosoma brucei, $T$. congolense et $T$. vivax (8). Les méthodes séro-immunologiques, par ailleurs, en dosant les anticorps, permettent de détecter un contact antérieur avec le parasite mais ne permettent pas de distinguer une infection active d'une infection guérie spontanément ou traitée.

L'introduction récente du test de détection des antigènes circulants pallie ces difficultés : il signe une infection active (présence d'antigènes) et est spécifique par identification du ou des parasites en cause grâce à l'utilisation d'anticorps monoclonaux (5). Une fois les réactifs fournis, le test est simple à mettre en oeuvre. Grâce à ces avantages, ce test est appelé à devenir la référence pour le diagnostic et pour les enquêtes épidémiologiques.

Ce test a été évalué au Centre de recherches sur les trypanosomoses animales (CRTA) lors d'infections expérimentales ou à l'occasion d'enquêtes sur le terrain. Les réactifs (anticorps monoclonaux et conjugués) ont été gracieusement fournis par I'"International Laboratory for Research on Animal Diseases" (ILRAD, Nairobi, Kenya).

\section{MATÉRIEL ET MÉTHODES}

Les anticorps monoclonaux contre $T$. brucei, $T$. congolense et $T$. vivax et les conjugués correspondants ont été utilisés suivant le protocole fourni par l'ILRAD avec les réactifs.

\section{Les sérums}

Les sérums négatifs proviennent de bovins français et ont été fournis gracieusement par le Laboratoire national de pathologie bovine de Lyon.

Les sérums d'infections expérimentales proviennent de chèvres et de moutons infectés soit par $T$. congolense (stock Karankasso/83/CRTA/57), soit par T. vivax (stock Satiri/87/CRTA134). Les animaux, traités au Bérénil ${ }^{N D}$ à leur arrivée au laboratoire et mis en observation 1 à 2 mois avant expérimentation, ont été infectés mécaniquement à la seringue à raison de $10^{6}$ trypanosomes par ani- 
mal. lis ont été séparés en fonction de leur espèce (mouton ou chèvre), de leur race (Djallonké ou Sahélien) et de l'infection ( $T$. congolense, $T$. vivax), soit 8 groupes différents. Les 4 groupes d'animaux Djallonké comprennent 6 individus chacun, et les 4 groupes d'animaux Sahélien comprennent 4 individus chacun. La parasitémie de ces animaux a été examinée tous les jours et exprimée suivant un système de score (4). Des échantillons de sang jugulaire ont été prélevés tous les 3 à 4 jours pour la sérologie. La période d'observation a duré 84 jours.

Les sérums d'infections naturelles proviennent de bovins prélevés à l'occasion d'un suivi sanitaire en pays Lobi (Burkina Faso). II s'agit d'une zone à forte pression de glossines.

Tous les sérums ont été conservés congelés à - $20^{\circ} \mathrm{C}$ jusqu'au moment de leur utilisation. La réaction ELISA a été réalisée dans des plaques Dynatech Microtiter ${ }^{N D}$ (lot Immulon $\mathrm{M} 129 \mathrm{~B})^{*}$ et la lecture faite avec un lecteur Titertek Multiskan Plus ${ }^{N D^{*}}$ à la longueur d'onde $405 \mathrm{~nm}$.

\section{RÉSULTATS}

\section{Sérums prélevés en France}

Quarante sérums de bovins, élevés hors de tout contact avec les glossines, ont été testés vis-à-vis des 3 anticorps monoclonaux. Les moyennes des densités optiques (DO) observées ont été : 0,007 $\pm 0,004,0,009 \pm$ 0,004 et $0,007 \pm 0,003$ pour $T$. congolense, $T$. vivax et $T$. brucei, respectivement.

\section{Sérums provenant d'infections expérimentales faites au CRTA}

Les résultats sont regroupés dans les figures 1 et 2 , correspondant aux 8 groupes. Pour chaque groupe, sont données les moyennes des DO observées et les moyennes des scores de parasitémie. Les parasitémies indiquées sur les figures sont celles qui ont été observées le jour du prélèvement pour détection des antigènes. Les animaux de race Sahélienne présentent des pics de parasitémie plus élevés que les animaux de race Djallonké considérés trypanotolérants ; ceci est particulièrement net lors des infections à $T$. vivax.

Les cinétiques d'antigènes circulants sont étudiées de manière homologue : les anticorps monoclonaux anti- $T$. congolense et anti-T.vivax ont été utilisés, respectivement, pour les infections correspondantes.

Lors des infections à $T$. congolense (fig. 1), les antigènes circulants sont aisément détectables avec des valeurs de DO supérieures à 0,050 (seuil de positivité indiqué par I'ILRAD). Dans le cas des moutons Sahélien (fig. 1c), la détection des antigèns circulants a précédé de 3 à 4 jours la détection des parasites ; dans les autres cas, la détection des antigènes était simultanée ou postérieure à celle des parasites. Pour les moutons Djallonké (fig. 1a), l'antigénémie était positive au jour 20 après l'infection, alors que la parasitémie était négative ; l'inverse a été observé au jour 43 pour les chèvres Sahélienne (fig. 1d). Pendant toute la durée d'observation, 55,1 p. 100 des déterminations de la parasitémie effectuées furent trouvées positives alors que 63,2 p. 100 des tests d'antigénémie le furent (tabl. I).

TABLEAU I Infections à $\mathrm{T}$. congolense : comparaison des pourcentages de positivité obtenus par détection des parasites, des antigènes circulants et en combinant les deux tests.

\begin{tabular}{|c|c|c|c|c|c|}
\hline & $\begin{array}{l}\text { Moutons } \\
\text { Djallonké }\end{array}$ & $\begin{array}{l}\text { Chèvres } \\
\text { Djallonké }\end{array}$ & $\begin{array}{l}\text { Moutons } \\
\text { Sahélien }\end{array}$ & $\begin{array}{c}\text { Chèvres } \\
\text { Sahélienne }\end{array}$ & Total \\
\hline $\begin{array}{l}\text { Parasitémies } \\
\text { positives }\end{array}$ & $\begin{array}{c}50,20 \text { p. } 100 \\
(492)\end{array}$ & $\begin{array}{c}50,81 \text { p. } 100 \\
(492)\end{array}$ & $\begin{array}{c}66,77 \text { p. } 100 \\
(316)\end{array}$ & $\begin{array}{c}57,86 \text { p. } 100 \\
(318)\end{array}$ & $\begin{array}{c}55,12 \text { p. } 100 \\
(1618)\end{array}$ \\
\hline $\begin{array}{l}\text { Antigénémies } \\
\text { positives }\end{array}$ & $\begin{array}{c}44,21 \text { p. } 100 \\
(95)\end{array}$ & $\begin{array}{c}81,31 \text { p. } 100 \\
(91)\end{array}$ & $\begin{array}{c}79,03 \text { p. } 100 \\
(62)\end{array}$ & $\underset{(65)}{50,76 p .100}$ & $\begin{array}{c}63,25 \text { p. } 100 \\
(313)\end{array}$ \\
\hline $\begin{array}{l}\text { Tests } \\
\text { combinés }\end{array}$ & $\begin{array}{c}70,52 \text { p. } 100 \\
(95)\end{array}$ & $\begin{array}{c}89,24 \text { p. } 100 \\
(91)\end{array}$ & 87,09 p. 100 & ${ }_{(65)}^{83,07 \text { p. } 100}$ & $\begin{array}{c}82,42 \text { p. } 100 \\
(313)\end{array}$ \\
\hline
\end{tabular}

( ): Nombre d'examens réalisés.

* Poly Labo, BP 36, 67023 Strasbourg cedex, France. 

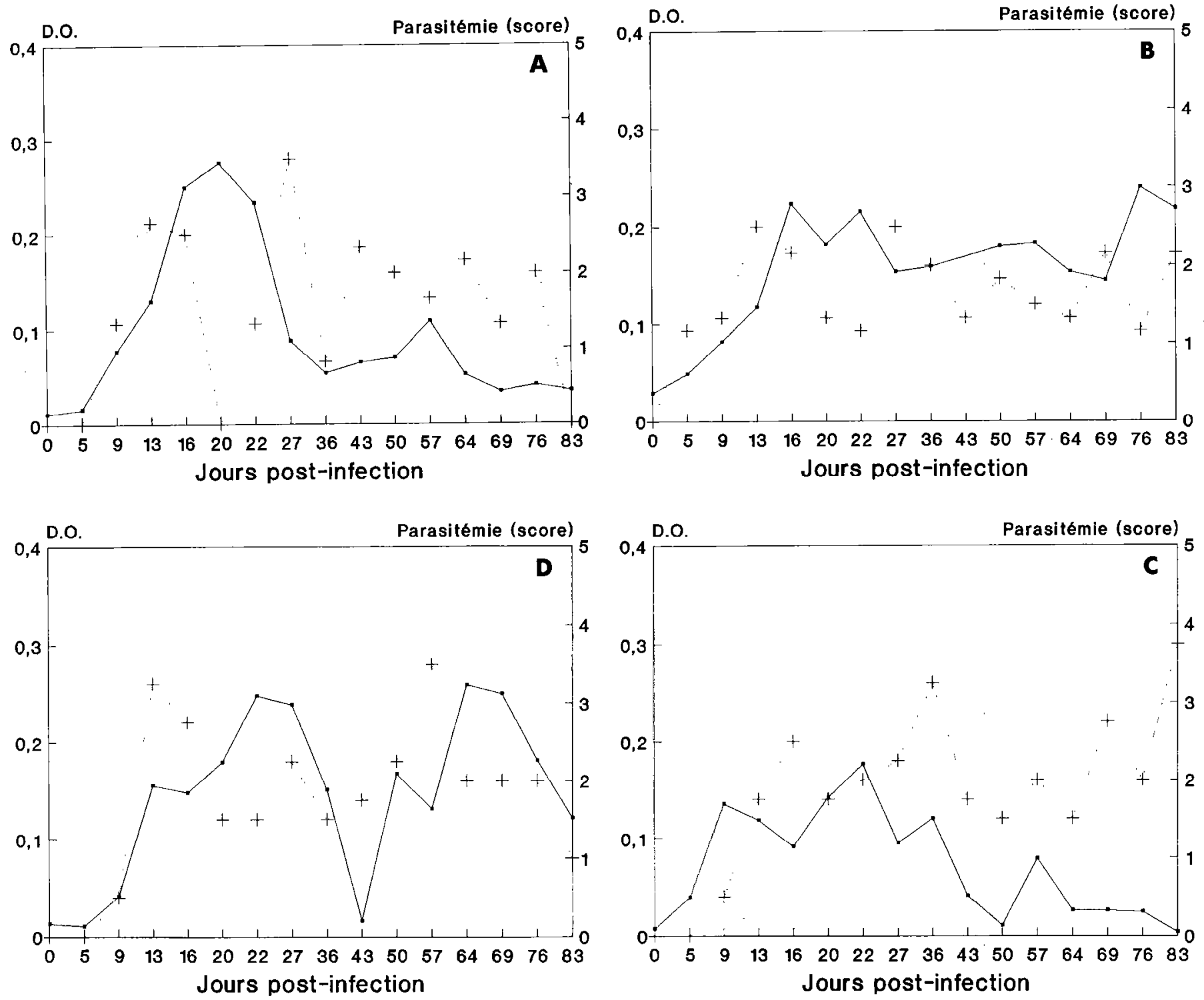

+ Moyennes des parasitémies

- Moyennes des DO

Figure 1 : Cinétique des antigènes circulants de T. congolense, en parallèle avec les parasitémies observées. a) moutons Djallonké (6 animaux) ; b) chèvres Djallonké (6); c) moutons Sahélien (4); d) chèvres Sahélienne (4).

Lors des infections à T.vivax (fig. 2), les antigènes circulants furent très difficiles à mettre en évidence. Dans seulement 9,9 p. 100 des cas observés, les DO obtenues furent supérieures à 0,050 alors que 48,6 p. 100 des parasitémies furent positives (tabl. II). A noter, pour les chèvres Sahélienne (fig. $2 \mathrm{~d}$ ), la mort de trois chèvres sur quatre au jour 63 , ce qui montre leur grande sensibilité à ce type d'infection.

L'association des deux tests (positivité de la parasitémie ou de l'antigénémie) est indiquée dans les tableaux I et II.
Pour les infections à $T$. congolense, 82,4 p. 100 des tests réalisés sont alors positifs et 52,8 p. 100 pour les infections à T.vivax.

\section{Sérums provenant d'infections naturelles}

Parmi les milliers de sérums prélevés à l'occasion d'un suivi sanitaire en pays Lobi (Burkina Faso), 40 ont été choisis, correspondant à des animaux détectés parasitologiquement positifs sur le terrain : 20 à $T$. congolense et 20 à $T$. vivax. Les résultats obtenus avec le test de détection des antigènes circulants sont regroupés dans le tableau III. 
A.B. Kanwe 7. Bengaly D. Saulnier G. Duvallet
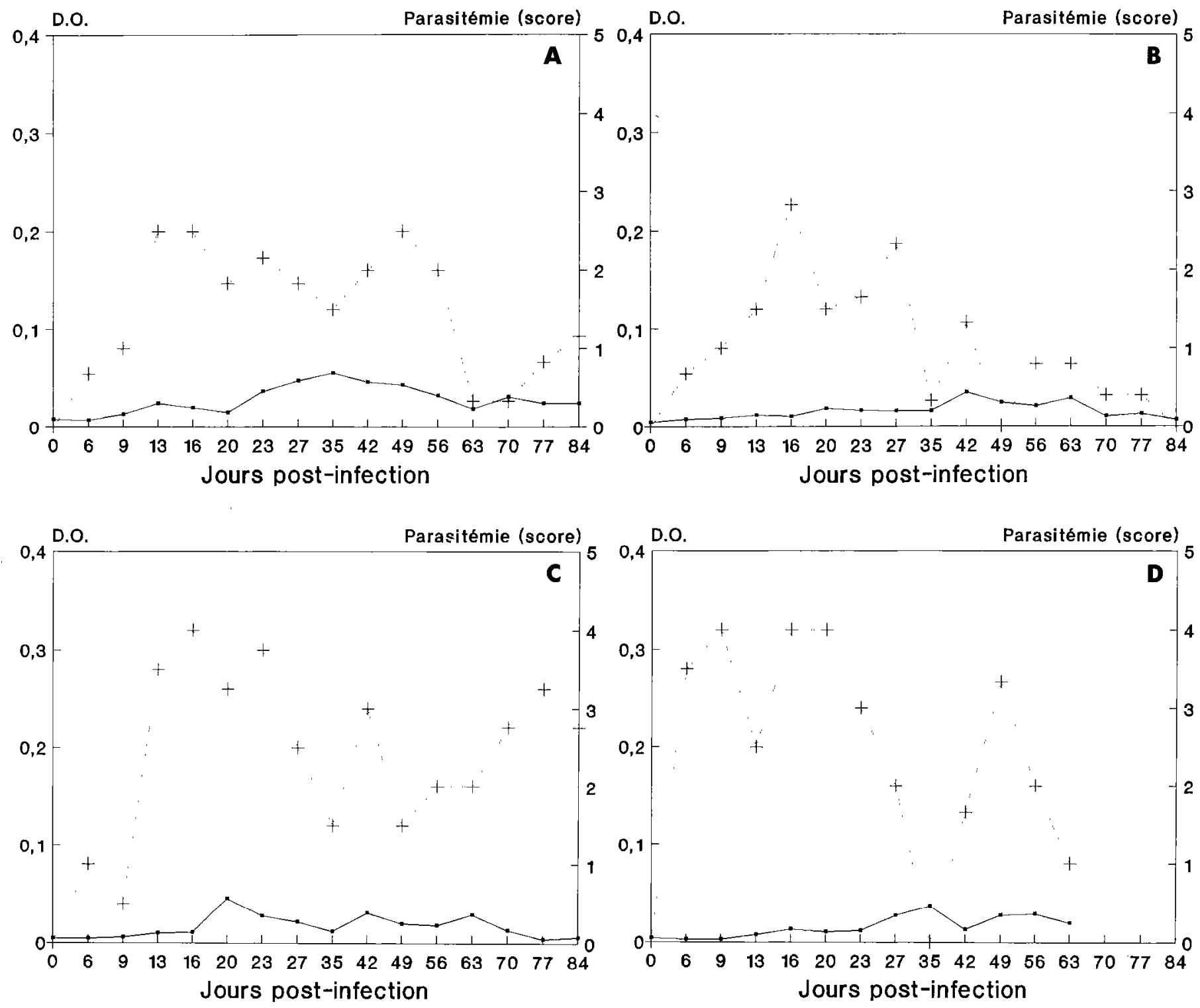

+ Moyenne des parasitémies

- Moyennes des no

Figure 2 : Cinétique des antigènes circulants de T. vivax, en parallèle avec les parasitémies observées. a) moutons Djallonké (6 animaux) ; b) chèvres Djallonké (6); c) moutons Sahélien (4); d) chèvres Sahélienne (4).

TABLEAU II Infections à T. vivax : comparaison des pourcentages de positivité obtenus par détection des parasites, des antigènes circulants et en combinant les deux tests.

\begin{tabular}{|c|c|c|c|c|c|}
\hline & $\begin{array}{l}\text { Moutons } \\
\text { Djallonké }\end{array}$ & $\begin{array}{l}\text { Chèvres } \\
\text { Djallonké }\end{array}$ & $\begin{array}{l}\text { Moutons } \\
\text { Sahélien }\end{array}$ & $\begin{array}{l}\text { Chèvres } \\
\text { Sahélienne }\end{array}$ & Total \\
\hline $\begin{array}{l}\text { Parasitémies } \\
\text { positives }\end{array}$ & $\begin{array}{c}41,56 \text { p. } 100 \\
(498)\end{array}$ & $\begin{array}{c}29,45 \text { p. } 100 \\
(455)\end{array}$ & $\begin{array}{c}74,05 \text { p. } 100 \\
(316)\end{array}$ & $\begin{array}{c}66,52 \text { p. } 100 \\
(233)\end{array}$ & $\begin{array}{c}48,60 \text { p. } 100 \\
(1502)\end{array}$ \\
\hline $\begin{array}{l}\text { Antigénémies } \\
\text { positives }\end{array}$ & $\begin{array}{c}17,30 \text { p. } 100 \\
(104)\end{array}$ & $\begin{array}{c}5,31 \text { p. } 100 \\
(94)\end{array}$ & $\begin{array}{c}6,55 \text { p. } 100 \\
(61)\end{array}$ & $\begin{array}{c}7,14 \text { p. } 100 \\
(42)\end{array}$ & $\begin{array}{c}9,96 \text { p. } 100 \\
(301)\end{array}$ \\
\hline $\begin{array}{l}\text { Tests } \\
\text { combinés }\end{array}$ & $\begin{array}{c}45,19 p .100 \\
(104)\end{array}$ & $\begin{array}{c}35,10 \text { p. } 100 \\
(94)\end{array}$ & $\begin{array}{c}77,04 \text { p. } 100 \\
(61)\end{array}$ & $\begin{array}{c}76,19 \text { p. } 100 \\
(42)\end{array}$ & $\begin{array}{c}52,82 \text { p. } 100 \\
(301)\end{array}$ \\
\hline
\end{tabular}

( ): Nombre d'examens réalisés. 
TABLEAU III Infections naturelles: moyennes des densités optiques observées chez des bovins trouvés infectés à T. congolense ou T. vivax sur le terrain.

\begin{tabular}{|l|c|c|}
\hline \multirow{2}{*}{$\begin{array}{c}\text { Anticorps monoclonaux } \\
\text { utilisés }\end{array}$} & \multicolumn{2}{|c|}{ Densité optique movenne } \\
\cline { 2 - 3 } & $\begin{array}{c}\text { Bovins infectés à } \\
\text { T. congolense (20) }\end{array}$ & $\begin{array}{c}\text { Bovins infectés à } \\
\text { T. vivax (20) }\end{array}$ \\
\hline Anti-T. congolense & $0,116 \pm 0,03$ & $0,050 \pm 0,030$ \\
Anti-T. vivax & $0,032 \pm 0,017$ & $0,011 \pm 0,028$ \\
Anti-T. brucei & $0,113 \pm 0,085$ & $0,057 \pm 0,035$ \\
\hline
\end{tabular}

( ): Nombre de sérums testés.

La moyenne des DO des sérums des animaux parasités par $T$. congolense était de $0,116 \pm 0,030$ avec le monoclonal anti- $T$. congolense, de $0,032 \pm 0,017$ avec le monoclonal anti- $T$. vivax et de $0,113 \pm 0,085$ avec le monoclonal anti- $T$. brucei.

Pour les animaux parasités par $T$. vivax, la moyenne des DO était de $0,050 \pm 0,030$ avec le monoclonal anti- $T$. congolense, de $0,011 \pm 0,028$ avec le monoclonal anti- $T$. vivax et de $0,057 \pm 0,035$ avec le monoclonal anti-T. brucei.

Parmi les 20 sérums infectés à $T$. congolense, 16 ont donné une DO supérieure à 0,050 en réaction homologue. Parmi les sérums infectés à $T$. vivax, un seul sur 20 a donné une DO supérieure à 0,050 en réaction homologue.

\section{DISCUSSION}

Les sérums prélevés sur des bovins en France étaient négatifs lors de la détection des antigènes circulants par ELISA. Les DO observées $(0,007$ à 0,009$)$ étaient très inférieures au seuil recommandé par l'ILRAD pour la positivité $(0,050)$.

Les résultats provenant de chèvres et de moutons infectés expérimentalement par $T$. congolense ou $T$. vivax présentent une variabilité inattendue. Si l'on retient comme sensibilité du test le nombre de vrais positifs sur la somme des vrais positifs et des faux négatifs (2), la détection des antigènes circulants par ELISA a une sensibilité de 63,2 p. 100 pour les animaux infectés par $T$. congolense et de 9,9 p. 100 seulement pour les animaux infectés par $T$. vivax.

MASAKE et NANTULYA (2) ont étudié la sensibilité du test lors d'infections expérimentales de chèvres et de bovins à $T$. congolense. Ils ont obtenu des sensibilités de 94,3 p. 100 chez les chèvres et de 82,5 p. 100 chez les bovins mais leur période d'observation était beaucoup plus longue : 3 ans pour les chèvres et 2 ans pour les bovins. Notre période d'observation fut de 84 jours seulement, correspondant à la première moitié de la phase parasitémique de leurs chèvres. Or, les résultats de ces auteurs montrent clairement que l'intérêt du test se situe au niveau de la longue phase aparasitémique : plus de 130 semaines sans parasite détectable mais avec une antigénémie positive. C'est au cours de la phase aiguë de l'infection qu'ils ont observé le plus de faux négatifs. Ils ont d'autre part réalisé des prélèvements mensuels chez les chèvres, alors que l'on a fait des prélèvements quotidiens pour la parasitémie et bihebdomadaires pour l'antigénémie. Nos résultats montrent l'intérêt de l'association des deux tests pendant la phase de parasitémie aiguë. Pour les infections à $T$. congolense, la sensibilité de la détection passe en effet à 82,4 p. 100 .

Les résultats des infections expérimentales à $T$. vivax montrent une très faible détection des antigènes circulants. Sur 301 tests réalisés, seuls 9,9 p. 100 ont donné une DO supérieure à 0,050 . Cependánt, les animaux ont montré une parasitémie importante pendant toute la durée d'observation : 48,6 p. 100 des 1502 examens parasitologiques étaient positifs. Un résultat similaire se retrouve avec les sérums de bovins naturellement infectés. La moyenne des DO des 20 sérums provenant d'animaux découverts infectés à $T$. vivax sur le terrain était de 0,011 , équivalente à celle des sérums d'animaux sains.

II semble que l'anticorps monoclonal anti- $T$. vivax ne reconnaisse pas, ici, les antigènes des animaux infectés, naturellement ou expérimentalement, à $T$. vivax. Ou bien on peut supposer que les réactifs étaient périmés ou que les animaux n'avaient pas d'antigènes circulants.

Des résultats positifs ont pourtant été obtenus avec cet anticorps monoclonal à l'ILRAD avec des bovins infectés expérimentalement par un stock de $T$. vivax originaire du Nigeria (5). NATULYA et al (6) avaient également montré que cet anticorps monoclonal, préparé par immunisation des souris avec un clone dérivé d'un stock du Nigeria, réagissait avec des stocks du Kenya et d'Ouganda.

Pour tester si les réactifs étaient encore efficaces, on a analysé un grand nombre de sérums de la sérothèque et certains donnaient des valeurs de DO élevées avec l'anticorps monoclonal anti-T. vivax (résultats non présentés). Ces sérums provenaient d'animaux prélevés en zone d'infection mais ne présentant pas de parasites au moment du prélèvement ; certains de ces animaux avaient été traités très récemment. NANTULYA et LINDQVIST (5) ont en effet montré la persistance des antigènes circulants dans la circulation, au moins deux semaines après le traitement. D'autre part, la conservation des sérums au congélateur à $-20^{\circ} \mathrm{C}$, pendant une période inférieure à un an comme dans cette expérimentation, ne dégrade pas les antigènes. Ceci est montré par les résultats obtenus avec les infections à $T$. congolense et par d'autres résultats obtenus avec des sérums conservés ainsi plus de trois ans.

II semble donc, en particulier pour $T$. vivax, que le test de détection des antigènes circulants ne donne que des résultats faiblement positifs pendant la période de parasitémie aiguë. Ceci confirmerait son intérêt pendant les 
périodes aparasitémiques et l'intérêt d'associer cette détection à la recherche des parasites pour une bonne sensibilité.

II est nécessaire de remarquer que la valeur élevée de DO de 0,050 avaient été retenue par l'ILRAD comme seuil de positivité pour diminuer le nombre de faux positifs. Cette valeur correspond aussi au seuil de visibilité à l'oeil nu. Pour les infections à T. vivax, il serait plus réaliste de définir une valeur plus faible, soit à partir des sérums de préinfection, soit à partir d'une population animale locale élevée dans une zone non infestée. Mais, dans ce cas, le test n'est plus lisible à l'oeil nu et nécessite un lecteur ELISA.

Des évaluations complémentaires sont encore nécessaires sur le terrain dans différentes conditions et le traitement des sérums pour "casser" les immun-complexes pourrait aussi augmenter la sensibilité du test (3).

\section{CONCLUSION}

Le test de détection des antigènes circulants à l'aide d'anticorps monoclonaux anti- $T$. congolense, anti- $T$. vivax et anti- $T$. brucei a été évalué au CRTA dans des condi-

KANWE (A.B.), BENGALY (Z.), SAULNIER (D.), DUVALLET (G.). Evaluation of the antigen delection lest for the diagnusis of trypanosomes using monoclonal antibodies. Experimental and natural infections. Revue Élev. Méd. vét. Pays trop., 1992, 45 (3-4) : 265-271

An antigen detection ELISA for the diagnosis of trypanosomes was recently proposed by NANTULYA and LINDQVIST (1989). Based on species-specific monoclonal antibodies, this test could be used to diagnose a current infection and to identify the causing trypanosomes. The test was evaluated at CRTA during experimental infections in small ruminants and with sera from naturally infected cattle, thanks to reagents supplied by the International Laboratory for Research on Animal Diseases (ILRAD). Sera from cattle sampled in France were also tested. Cattle sera from France gave optical densities (OD) from 0.007 to 0.009 with three monoclonal antibodies against $T$. congolense, $T$. vivax and $T$. brucei. These $O D$ values were well below 0.050 , which is considered as a positive threshold OD reading. In the small ruminant experimental infections, the sensitivity of the test was $63.2 \%$ for $T$. congolense-infected animals and $9.9 \%$ for $T$. vivaxinfected animals. The sensitivity of parasitological tests was 55.1 and $48.6 \%$, respectively. The combination of the antigen- and parasitedetection tests increased the sensitivity to 82.4 and $52.8 \%$, respectively. Means of $O D$ values, with the naturally infected cattle sera, were $0.116 \pm 0.030$ for $T$. congolense, and $0.011 \pm 0.028$ for $T$. vivax -infected animals. Sixteen out of $20 \mathrm{~T}$. congolense-infected sera (sensitivity of $80 \%$ ) and one out of $20 \mathrm{~T}$, vivax-infected sera (sensitivity of $5 \%$ ) gave an $O D$ value exceeding 0.050 . The determination of a threshold OD reading lower than 0.050 would greatly improve the sensitivity of the test. This determination could either be done by studying the preinfection sera or a local population of animals living in an area free from trypanosomosis. Our results have been obtained during the acute phase of infection. Results from ILRAD show that this test is much more reliable during the aparasitaemic chronic phase. Other evaluations for epidemiological surveys in the field are being carried out at CRTA. Key words : Trypanosoma congolense - Trypanosoma vivax - Trypanosoma brucei - Trypanosomosis - Diagnosis - Antigen detection test - ELISA test - Monoclonal antibodies - Burkina Faso. tions d'infections exprimentales chez des petits ruminants, et dans des conditions d'infections naturelles chez des bovins. En retenant la valeur de DO de 0,050 comme seuil de positivité, ce test a montré une bonne sensibilité lors des infections à $T$. congolense, mais une sensibilité très faible lors des infections à $T$. vivax. Nos observations ont été réalisées essentiellement pendant les phases de parasitémies aiguës. Les résultats présentés montrent l'intérêt d'associer la détection des parasites et la détection des antigènes circulants pendant ces périodes. La comparaison avec les résultats de la littérature montre que la sensibilité de ce test augmente considérablement pendant les phases de parasitémies chroniques, lorsque les parasites ne sont plus détectables.

\section{REMERCIEMENTS}

Nous remercions vivement l'ILRAD et le Dr V.M. NANTULYA pour le don de leurs réactifs : anticorps monoclonaux et conjugués, le Laboratoire national de pathologie bovine de Lyon (Dr SAVEY) pour le don de sérums de bovins prélevés en France, le professeur UILENBERG (CIRADEMVT) et le Dr RACHAEL A. MASAKE (ILRAD) pour la lecture du manuscrit et leurs critiques constructives.

KANWE (A.B.), BENGALY (Z.), SAULNIER (D.), DUVALLET (G.). Evaluación de un test de detección de antígenos circulantes de tripanosomas, mediante anticuerpos monoclonales. Infecciones experimentales y naturales. Revue Élev. Méd. vét. Pays trop., 1992, 45 (3-4) : 265-271

Recientemente, NANTULYA y LINDQVIST (1989), propusieron un test ELISA para la detección de antígenos circulantes para el diagnóstico de tripanosomosis animales. Gracias al uso de anticuerpos monoclonales espcíficos, este test debería permitir el diagnóstico de la infección activa y la identificación del (los) tripanosoma(s) responsable(s). Gracias a una donación de reactivos por parte del International Laboratory for Research on Animal Diseases (ILRAD), se pudo evaluar este test en el CRTA, tanto en infecciones experimentales en pequeños rumiantes, como en sueros bovinos colectados en el campo. Además e ésto, se obtuvieron densidades ópticas (DO) de 0,007 a 0,009 , obtenidas con anticuerpos monoclonales anti $T$. congolense, anti $T$, vivax y anti $T$. brucei, en 40 sueros bovinos colectados en Francia. Estas DO son bastante inferiores a 0,050, valor límite de positividad aceptada por el ILRAD. En las infecciones experimentales en pequeños rumiantes, la sensibilidad del test fue de $63,2 \mathrm{p} .100$ para los animales infectados con $T$. congolense y de $9,9 \mathrm{p}$. 100 para aquellos infectados con T. vivax. La sensibilidad de los exámenes parasitológicos fue dé 55,1 y 48,6 p. 100 respectivamente. El uso combinado de los dos tests, permitió aumentar la sensibilidad a 82,4 y 52,8 p. 100 respectivamente. En cuanto a los sueros bovinos infectados, colectados en condiciones de campo, se observaron densidades ópticas promedio, en reacción homóloga, de $0,116 \pm 0,030$ para $T$. congolense y de $0,011 \pm 0,028$ para $T$, vivax. De los 20 sueros infectados con $T$. congolense, 16 obtuvieron una DO superior a 0,050 (sensibilidad 80 p. 100) y de los 20 sueros animales infectados con $T$. vivax, solamente uno obtuvo una DO superior a 0,050 (sensibilidad 5 p. 100). EI establecimiento de un valor límite de DO inferior a 0,050 , basado en el analisis de sueros pre-infección, o en el estudio de animales provenientes de zonas no infectadas, podría permitir un aumento de la sensibilidad del test. Vale la pena señalar que éstos resultados fueron obtenidos durante la fase de infección aguda de los animales, principalmente en las infecciones experimentales. Los resultados presentados por el ILRAD demuestran que este test es mucho más eficaz durante las fases crónicas sin parasitosis. En este momento se llevan a cabo otros estudios en el CRTA, principalmente encuestas epidemiológicas en condiciones de campo. Palabras claves : Trypanosoma congolense - Trypanosoma vivax - Trypanosoma brucei. Tripanosomosis - Diagnóstico - Detección de antígenos circulantes - Test ELISA - Anticuerpos monoclonales - Burkina Faso. 


\section{BIBLIOGRAPHIE}

1. KRATZER (R.D.), ONDIEK (F.O.). The buffy coat double centrifugation technique, an improved method for the diagnosis of African trypanosomiasis. 20e réunion UUA/CSIR'TC, 10-14 avril 1989, Mombasa, Kenya. ( $\mathrm{N}^{\circ} 321$, liste provisoire des résumés).

2. MASAKE (R.A.), NANTULYA (V.M.). Sensitivity of an antigen detection enzyme immunoassay for diagnosis of Trypanosoma congolense infections in goats and cattle. J. Parasit., 1991, 77 (2):231-236.

3. MORE (S.J.), COPEMAN (D.B.). Antigen detection ELISAs : Pretreatment of serum to reduce interference by specific host antibodies. Trop. Med Parasit., 1991, 42 : 91-94.

4. MURRAY (M.), MURRAY (P.K.), McINTYRE (W.I.M.). An improved parasitological technique for the diagnosis of African trypanosomiasis. Trans. R. Soc. Trop. Med. Hyg., 1977, 71 : 325-326.

5. NANTULYA (V.M.), LINDQVIST (K.J.). Antigen-detection enzyme immunoassays for the diagnosis of Trypanosoma vivax, $T$. congolense, T. bruce i infections in cattle. Trop. Med. Parasit., 1989, 40 (3): 267-272.

6. NANTULYA (V.M.), MUSOKE (A.J.), RURANGIRWA (F.R.), SAIGAR (N.), MINJA (S.H.). Monoclonal antibodies that distinguish Trypanosoma congolense, Trypanosoma vivax and Trypanosoma brucei. Parasit. Immun., 1987, 9 (4): $421-431$.

7. PARIS (I.), MURRAY (M.), McODIMBA (F.). A comparative evaluation of the parasitological techniques currently available for the diagnosis of African trypanosomiasis in cattle. Acta Tropica, 1982, $39: 307-316$.

8. VERY (P.), BOCQUENTIN (R.), DUVALLET (G.). Sensibilité de la double microcentrifugation pour la recherche des trypanosomes. Revue Élev. Méd. vét. Pays trop., 1990, 43 (3) : 325-329. 